\title{
Caso clínico: Rabdomiolisis secundaria al consumo de cocaína
}

\author{
J. I. DOMÍNGUEZ1', J. M. CAMPERO², M. REYES', M. F. SAN MARTÍN ${ }^{4}$
}

\begin{abstract}
Case report: Rabdomuolisis after cocaine consumption

Introduction: The multiple causes of rhabdomyolysis can be separated into 5 categories according to the myocyte damage mechanism: hypoxic, physical, chemical and biological. However, a cause will not always be identified. The consumption of illicit drugs / alcohol has been identified as the first cause, followed by medication, trauma, immobility and extenuating exercise. The chemical causes represent up to $80 \%$ of cases of rhabdomyolysis. This category includes medications, alcohol and drugs, where cocaine stands out. Cocaine is a strong stimulant used mainly as a recreational drug that has considerably increased its consumption in the country so it should be considered. Next, we present the case of a male patient with acute renal failure secondary to rhabdomyolysis due to cocaine use. The physiopathology, diagnostic process and current management will be discussed according to the latest guidelines.
\end{abstract}

\section{RESUMEN}

Introducción: Las múltiples causas de Rabdomiolisis se pueden separar en 5 categorías según el mecanismo de daño del miocito: Hipóxicas, físicas, químicas, biológicas e idiopáticas. Como primera causa se describe el consumo de drogas ilícitas/alcohol, seguido por medicamentos, trauma, inmovilidad y ejercicio extenuante. Las causas químicas representan hasta el $80 \%$ de los casos de rabdomiolisis. En esta categoría se encuentran los medicamentos, alcohol y drogas donde destaca la cocaína. Se presenta el caso de un paciente masculino que cursa con insuficiencia renal aguda secundario a una rabdomiolisis por consumo de cocaína.

Facultad de Medicina Pontificia Universidad Católica de Chile.

Facultad de Medicina Universidad del Desarrollo-Clínica Alemana de Santiago.

Residente Medicina Interna Pontificia Universidad Católica de Chile.

\section{Key words:}

Rhabdomyolysis, Acute renal failure, cocaine, creatinkinase.
Palabras clave:

Rabdomiolisis, Falla renal aguda, cocaína, creatinkinasa.

Fecha de recepción: 25 de enero de 2018

Fecha de aceptación: 19 de febrero de 2018

\section{ORCID}

https://orcid.org/0000-0002-4203-6146

\section{Correspondencia:}

Dra. Magdalena Reyes

Email: mpreyes4@uc.cl 


\section{Resumen del caso}

P aciente masculino de 35 años, sin antecedentes mórbidos, ingresa por compromiso de conciencia y agitación psicomotora y con historia de consumo de alcohol y cocaína en días previos.

Al ingreso en el laboratorio general destacan pruebas hepáticas alteradas, falla renal aguda, alteraciones hidroelectrolíticas y elevación severa de CKtotal.

Se inicia manejo de falla renal aguda y se extiende el estudio etiológico descartándose causa neurológica del compromiso de conciencia y serología hepática negativa. Evoluciona anúrico, hipertenso, polipneico, mala mecánica ventilatoria, y sopor profundo por lo que se traslada a UCl y es conectado a ventilación mecánica indicándose diálisis de emergencia. Es extubado a las 48 horas y luego trasladado a sala, donde evoluciona favorablemente, pero se mantiene anúrico por lo que requiere diálisis trisemanal.

\section{Discusión}

La rabdomiolisis es un síndrome potencialmente mortal caracterizado por daño al miocito y liberación del material intracelular tóxico a la circulación sistémica. Dentro de las sustancias liberadas destacan electrolitos, mioglobina, creatin-quinasa (CK), aldolasa y lactato deshidrogenasa (LDH). Su desarrollo se asocia a enfermedades, toxinas, medicamentos e injurias[1].

El cuadro clínico es variado presentándose de forma asintomática hasta llegar a ser potencialmente mortal, con elevación extrema de CK, alteraciones hidroelectrolíticas e insuficiencia renal aguda (IRA)[2] en el $10-50 \%$ de los casos[3].

En condiciones normales la mioglobina circula unida a proteínas. Su concentración no supera los 0,003 mg/dL. Si se dañan más de $100 \mathrm{mg}$ de músculo, la cantidad de mioglobina plasmática sobrepasa la capacidad de unión a proteínas y filtra por el glomérulo[1],[3].

Los mecanismos involucrados en la falla renal son precipitado de mioglobina en los glomérulos; formación de cilindros que causan obstrucción de los túbulos renales, secundario a la interacción de la mioglobina con la proteína Tamm-Horsfall; efecto citotóxico directo; vasoconstricción renal[4],[5].

Las causas de Rabdomiolisis se pueden separar en 5 categorías según el mecanismo de daño del miocito: Hipóxicas, físicas, químicas, biológicas e idiopáticas.

En un estudio que involucró a 475 pacientes hospitalizados por rabdomiolisis, se identificó como

\begin{tabular}{|c|c|c|}
\hline \multicolumn{3}{|c|}{$\begin{array}{c}\text { Tabla 1. Laboratorio de ingreso el 03/07/2017 } \\
\text { donde se evidencian los hallazgos descritos en la } \\
\text { presentación del caso }\end{array}$} \\
\hline Examen & Valor & Unidad \\
\hline Albúmina & 4,3 & $\mathrm{~g} / \mathrm{dL}$ \\
\hline Bilirrubina total & 1,36 & $\mathrm{mg} / \mathrm{dL}$ \\
\hline Fosfatasa alcalina & 145 & $\mathrm{U} / \mathrm{L}$ \\
\hline GOT & 4.794 & $U / L$ \\
\hline GPT & 2.112 & $\mathrm{U} / \mathrm{L}$ \\
\hline INR & 1,53 & \\
\hline Protrombina\% & $61 \%$ & \\
\hline $\begin{array}{l}\text { Creatinkinasa } \\
\text { total }\end{array}$ & 249.900 & $\mathrm{mg} / \mathrm{dL}$ \\
\hline Creatinkinasa MB & 4.540 & $\mathrm{mg} / \mathrm{dL}$ \\
\hline Creatinemia & 6,11 & $\mathrm{mg} / \mathrm{dL}$ \\
\hline Nitrógeno uréico & 46 & $\mathrm{mg} / \mathrm{dL}$ \\
\hline $\mathrm{Na}+$ & 128 & $\mathrm{mEq} / \mathrm{L}$ \\
\hline $\mathrm{K}+$ & 6,2 & $\mathrm{mEq} / \mathrm{L}$ \\
\hline $\mathrm{Cl}-$ & 98 & $\mathrm{mEq} / \mathrm{L}$ \\
\hline Calcio & 5 & $\mathrm{mg} / \mathrm{dL}$ \\
\hline Fósforo & 7,3 & $\mathrm{mg} / \mathrm{dL}$ \\
\hline Glóbulos blancos & 12.440 & células/mm³ \\
\hline $\mathrm{pH}$ & 7,3 & \\
\hline PCO2 & 36 & $\mathrm{mmHg}$ \\
\hline Bicarbonato & 17,4 & $\mathrm{mEq} / \mathrm{L}$ \\
\hline Exceso de base & -8 & $\mathrm{mmol} / \mathrm{L}$ \\
\hline
\end{tabular}

primera causa el consumo de drogas ilícitas/alcohol, seguido por medicamentos, trauma, inmovilidad y ejercicio extenuante (Tabla 1). La cocaína es un fuerte estimulante utilizado principalmente como droga recreativa que ha aumentado considerablemente su consumo. Según el último reporte mundial de la Oficina de las Naciones Unidas contra la Droga y el Delito 2015 , sitúa a Chile como el tercer país con mayor consumo de cocaína en América. Su uso aumenta el riesgo de sufrir accidentes cerebrovasculares, infarto al miocardio, paro cardiorespiratorio y rabdomiolisis entre otros.

El músculo esquelético es más propenso al daño hipóxico y al compromiso vascular debido a su ubicación periférica. Esto se puede evidenciar en procedimientos quirúrgicos prolongados y en casos de trombosis venosa o arterial.

Las causas físicas de rabdomiolisis pueden de- 
Tabla 2. Etiología de Rabdomiolisis. Condiciones asociadas a rabdomiolisis según estudio realizado a 475 pacientes

\begin{tabular}{lcccc}
\hline \multicolumn{1}{c}{ Categoria } & n de pacientes & Hombres (\%) & IRA & Muertes \\
Drogas ilicitas/Alcohol & 163 & 82 & 106 & 5 \\
Drogas Médicas & 54 & 72 & 26 & 1 \\
Enfermedad muscular & 49 & 37 & 2 & 1 \\
Trauma & 42 & 58 & 19 & 2 \\
NMS & 38 & 74 & 13 & 3 \\
Idiopático & 34 & 50 & 12 & 1 \\
Convulsiones & 32 & 75 & 11 & 1 \\
Inmovilidad & 21 & 52 & 6 & 1 \\
Causa metabólica & 9 & 56 & 1 & 0 \\
VIH/SIDA & 9 & 67 & 2 & 0 \\
Otra & 7 & 43 & 4 & 0 \\
UCl miopatia & 4 & 75 & 0 & 0 \\
Hipertermia maligna & 3 & 100 & 3 & 0 \\
Ejercicio & 3 & 100 & 2 & 0 \\
Calor/Deshidratación & 3 & 67 & 2 & 17 \\
Mieloma múltiple & 2 & 100 & 1 & \\
Hipotermia & 2 & 100 & 226 & \\
Total & 475 & 71,1 & \\
\hline
\end{tabular}

berse a traumatismos como lesión contusa, aplastamiento, electrocución o quemaduras, pero también al esfuerzo muscular excesivo en actividades deportivas, convulsiones o hipertermia[6].

Las causas químicas representan hasta el $80 \%$ de los casos de rabdomiolisis[7]. En esta categoría se encuentran los medicamentos (quetiapina, estatinas, amiodarona, warfarina, benzodiacepinas, entre otros), alcohol y drogas ilícitas (cocaína, heroína, metanfetaminas, NMDA-éxtasis)[4]. Éstos aumentan la actividad muscular, precipitan convulsiones, causan hipertermia o vasoconstricción[1] (Tabla 1)[8].

Del punto de vista anestésico es importante considerar que muchos de los fármacos utilizados pueden desencadenar rabdomiolis, por ejemplo, los halogenados y relajantes musculares como la succinilcolina. Y además debe considerarse que cada vez más pacientes son consumidores de cocaína habituales y son sometidos a procedimientos quirúrgicos.

Se ha descrito también rabdomiolisis secundaria al uso de propofol en infusión por períodos largos de tiempo, en contexto de un Síndrome por infusión de propofol. Caracterizado por desarrollo de acidosis láctica severa, rabdomiolisis, falla cardiaca, hepática y renal[11].

Finalmente, dentro de las causas biológicas se encuentran infecciones bacterianas principalmente por gram positivos e infecciones virales como Influenza $A$, $B, \mathrm{VIH}$ y Enterovirus. También se han reportado casos debido a toxinas de picaduras de abeja, hormiga y escorpión[1].

Presentación variada por lo que requiere un alto índice de sospecha[12]. Se describe la tríada clásica de mialgias, debilidad muscular y orina oscura. En el $50 \%$ de los casos las mialgias se localizan en grupos musculares proximales, sin embargo más de la mitad de los pacientes no reportan dolor[4]. En casos severos pueden aparecer taquicardia, náuseas, vómitos y fiebre.

La rabdomiolisis se confirma con estudios de laboratorio que permiten un diagnóstico precoz. Niveles elevados de CK son marcadores[13]. Se eleva dentro de las primeras 12 horas posteriores a la injuria, alcanzando el peak a los 1-3 días, con descenso a los 3-5 días[14]. Niveles sobre $5.000 \mathrm{U} / \mathrm{L}$ son altamente sugerentes de rabdomiolisis[15], mientras que valores sobre $15.000 \mathrm{U} / \mathrm{L}$ se asocian a falla renal[12].

En orina el Dipstick es reactivo para mioglobina 
cuando sus niveles séricos superan los 0,3 mg/ ${ }^{12}$. Al examen microscópico se puede observar cilindros de mioglobina y cristales de ácido úrico. En el físico-químico el pH urinario es ácido[12] y el color marrón rojizo, lo que se ve cuando la mioglobina sérica supera los $100 \mathrm{mg} / \mathrm{L} 1$.

Su manejo consiste en una volemización precoz y agresiva, corrección del factor etiológico y manejo de las complicaciones, además de prevenir mayor daño muscular y la consecuente falla renal aguda que se relacionan a menor sobrevida[16]. Es importante la monitorización del débito urinario y controles seriados de laboratorio, incluyendo creatinina, electrolitos, albúmina y gases[8].

Se recomienda volemización vigorosa con solución fisiológica para mantener diuresis entre 200 a $300 \mathrm{ml} / \mathrm{h}$ hasta que los niveles de CK estén bajo 1.000
U/L[17]. Es importante el monitoreo continuo de signos vitales y débito urinario para evaluar respuesta. El uso de bicarbonato para alcalinizar la orina $(\mathrm{pH}>6,5)$ no tiene evidencia clara. El uso de Manitol tampoco tiene suficiente evidencia y no se recomienda de rutina[15].

Finalmente, la hemodiálisis es útil en casos de insuficiencia renal aguda asociada a hiperkalemia severa, síndrome urémico, acidosis e hipervolemia refractarias a terapia médica[18].

En conclusión la rabdomiolisis varía considerablemente según la causa y comorbilidades del paciente. La estadía hospitalaria y la mortalidad pueden llegar a triplicarse en aquellos que presentan insuficiencia renal aguda[19]. La mortalidad varía entre 1,7-46\% en las distintas series. Para su diagnóstico es fundamental la alta sospecha clínica.

\section{Referencias}

1. Khan F. Rhabdomyolysis: a review of the literature. The journal of medicine. 2009;67:272283.

2. Huerta-Alardin A, Varon J, Marik $P$. Bench-to-bedside review: rhabdomyolysis- an overview for clinicians. Critical Care. 2005, 9:158-169.

3. Grossman $\mathrm{R}$, Hamilton $\mathrm{R}$, Morse B, Penn A, Goldberg M: Nontraumatic rhabdomyolysis and acute renal failure. N Engl J Med 1974, 291:807-811.

4. Williams J, Thorpe C. Rhabdomyolysis. BJA Education. 2014;14:163-166.

5. Bosch X, Poch E, Grau J. Rhabdomyolysis and acute kidney injury. N Engl J Med. 2009;361:6272.

6. S. Guis, et al. Pathophysiology and clinical presentations of rhabdomyolysis. Joint Bone Spine 2005; (72): 382-391.

7. Richards J. Rhabdomyolysis and drugs of abuse. J Emerg Med. 2000;19:51-56.

8. Melli G, Chaudhry V, Cornblath
D. Rhabdomyolysis. An Evaluation of 475 Hospitalized Patients Medicine. 2005; (84):377-385.

9. Escobar J. Hipertermia Maligna. Rev Med Clin Condes. 2011; 22: 310-315.

10. Urwyler A, Deufel T, McCarthy T, West S. Guidelines for molecular genetic detection of susceptibility to malignant hyperthermia. $\mathrm{Br}$ J Anaesth 2001; 86: 283- 7.

11. Corbett SM, Montoya ID, Moore FA. Propofol-related infusion syndrome in intensive care patients. Pharmacotherapy. 2008;28:250-8.

12. Giannoglou $G$, Chatzizisis $Y$, Misirli G. The syndrome of rhabdomyolysis: Pathophysiology and diagnosis. European Journal of internal medicine. 2007; 18:90100.

13. Gabow PA, Kaehny WD, Kelleher SP. The spectrum of rhabdomyolysis. Medicine 1982;61:141-53.

14. Nemiroff L, Cormier S, LeBlanc C, Murphy N. Considering acute rhabdomyolysis in emergency department patients with cocaine ingestion. Canadian Family Physician. 2012;58:750-754.
15. Cervellin G, Comelli I, Lippi G. Rhabdomyolysis: historical background, clinical, diagnostic and therapeutic features. Clin Chem Lab Med 2010;48(6):749-56.

16. De Meijer AR, Fikkers BG, de Keijzer $M H$, et al. Serum creatine kinase as predictor of clinical course in rhabdomyolysis: a 5-year intensive care survey. Intensive Care Med 2003; 29: 1121-5.

17. Scharman EJ, Troutman WG. $C$ Prevention of kidney injury following rhabdomyolysis: a systematic review. The Annals of pharmacotherapy. 2013;47(1):90-105.

18. Mikkelsen TS, Toft P. Prognostic value, kinetics and effect of CVVHDF on serum of the myoglobin and creatine kinase in critically ill patients with rhabdomyolysis. Acta Anaesthesiol Scand. 2005;49(6):859.

19. Scharman EJ, Troutman WG. $C$ Prevention of kidney injury following rhabdomyolysis: a systematic review. The Annals of pharmacotherapy. 2013;47(1):90-105. 